Chem. 273:27765-27767.

10. Koike, H., et al. 1999. Membrane-anchored metalloprotease MDC9 has an alpha-secretase activity responsible for processing the amyloid precursor protein. Biochem. J. 343:371-375.

11. Hong, L., et al. 2000. Structure of the protease domain of memapsin 2 (beta-secretase) complexed with inhibitor. Science. 290:150-153.

12. Selkoe, D., and Kopan, R. 2003. Notch and Presenilin: regulated intramembrane proteolysis links development and degeneration. Annu. Rev. Neurosci. 26:565-597.

13. Nitsch, R.M., Slack, B.E., Wurtman, R.J., and Growdon, J.H. 1992. Release of Alzheimer amyloid precursor derivatives stimulated by activation of muscarinic acetylcholine receptors. Science. 258:304-307.
14. Furukawa, K, et al. 1996. Increased activity-regulating and neuroprotective efficacy of alphasecretase-derived secreted amyloid precursor protein conferred by a C- terminal heparin-binding domain. J. Neurochem. 67:1882-1896.

15. Meziane, H., et al. 1998. Memory-enhancing effects of secreted forms of the beta-amyloid precursor protein in normal and amnestic mice. Proc. Natl. Acad. Sci. U. S. A. 95:12683-12688.

16. Skovronsky, D.M., Moore, D.B., Milla, M.E., Doms, R.W., and Lee, V.M. 2000. Protein kinase C-dependent alpha-secretase competes with betasecretase for cleavage of amyloid-beta precursor protein in the trans-golgi network. J. Biol. Chem. 275:2568-2575.

17. Slack, B.E., Ma, L.K., and Seah, C.C. 2001. Constitutive shedding of the amyloid precursor protein ectodomain is up-regulated by tumour necrosis factor-alpha converting enzyme. Biochem. J. 357:787-794.

18. Hartmann, D., et al. 2002. The disintegrin/ metalloprotease ADAM 10 is essential for Notch signalling but not for alpha-secretase activity in fibroblasts. Hum. Mol. Genet. 11:2615-2624.

19. Peschon, J.J., et al. 1998. An essential role for ectodomain shedding in mammalian development. Science. 282:1281-1284

20. Moechars, D., et al. 1999. Early phenotypic changes in transgenic mice that overexpress different mutants of amyloid precursor protein in brain. J. Biol. Chem. 274:6483-6492.

21. Sahin, U, et al. 2004. Distinct roles for ADAM10 and ADAM17 in ectodomain shedding of six EGFR ligands. J. Cell. Biol. 164:769-779.

\title{
Sepsis: avoiding its deadly toll
}

Thomas Decker

Max F. Perutz Laboratories, University Departments at the Vienna Biocenter, Department of Microbiology and Genetics, University of Vienna, Vienna, Austria.

\begin{abstract}
Systemic bacterial infection may culminate in a frequently fatal septic shock syndrome. The underlying pathology is the result of an uncontrolled inflammatory response, stimulated by the pathogen and its products. Tolllike receptors (TLRs) are critically involved in sensing bacteria and, in the case of sepsis, stimulate a pathogenic response by the innate immune system. A new study reports a successful attempt to inhibit systemic inflammation in mice by disrupting the formation of complexes between Grampositive bacteria and their cognate receptor, TLR2 (see the related article beginning on page 1473).
\end{abstract}

Sepsis results from the inability of the immune system to limit bacterial spread during an ongoing infection. Massive bacterial load overrides the inhibitory mechanisms controlling inflammation. While normally helping to eradicate pathogens from a local infection of peripheral tissues, inflammation during sepsis develops into a systemic syndrome with multiple manifestations such as tissue injury, increased vascular permeability, and, ultimately, multi-organ failure and shock (1). Mortality rates of septic patients are high (up to $70 \%$ ), and the costs of treatment have been calculated to amount to more than $\$ 15$ billion per year in the US alone (2). Since antibiotics carry the risk of promoting the release of bacterial products and thus to exacerbate the shock syndrome during sepsis, the development of

Nonstandard abbreviations used: high-mobility group box 1 protein (Hmgb1); pathogen-associated molecular pattern (PAMP); toll-like receptor (TLR). Conflict of interest: The author has declared that no conflict of interest exists.

Citation for this article:

J. Clin. Invest. 113:1387-1389 (2004).

doi:10.1172/JCI200421819. alternative treatments and the improvement of current regimens for treating septic patients are of high priority.

\section{The septic shock syndrome and its mediators}

The list of suitable targets for interruption of the inflammatory cascade begins with the receptors involved in the binding and uptake of bacteria and their products by cells of the innate immune system (phagocytes, dendritic cells, vascular endothelial cells). It continues with the many proinflammatory molecules produced by the innate immune system during infection, cytokines and/or chemokines like TNF- $\alpha$, IL-1, and IL-8, lipid mediators, oxygen radicals, and tissue-damaging enzymes. Components of the activated complement and coagulation systems also promote inflammation and are further candidate targets for inhibitory drugs (Figure 1). Several novel therapeutic approaches have been tested in animal models of septic shock and also in clinical trials (3). Activated protein C, an inhibitor of clotting factors, reduces monocyte synthesis of proinflammatory cytokines and the interaction of phagocytes with the vascular endothelium and has been approved for clinical use (reviewed in ref. 3 ). The therapeutic approach reported in this issue of the JCI by Meng and colleagues explores the possibility of interrupting an initial step of septic inflammation, the interaction of Gram-positive bacteria or their cell wall components with toll-like receptor 2 (TLR2) (4).

\section{TLRs in innate immune responses to pathogens}

TLRs belong to the group of germlineencoded, nonclonal receptors, which is functionally defined by the ability to discriminate self from pathogen-associated molecular patterns (PAMPs) (5). Ten members of the TLR family in humans and 11 in rodents allow cells of the innate immune system to respond with appropriate intracellular signals to the presence of all groups of microorganisms (6, $7)$. TLR signaling stimulates cell-autonomous antimicrobial defense systems as well as the secretion of immunoregulatory substances, including many of the proinflammatory molecules listed above. Associations between the susceptibility to pathogens and defective or absent TLRs have been established by genetic linkage with inactivating mutations in humans (8), and by targeted disruption of th loci in mice (6). Further examples of immunological benefits resulting from TLR stimulation have been provided by the antiviral imidazoquinolines and guanosine analogues that exert their effects via TLRs 


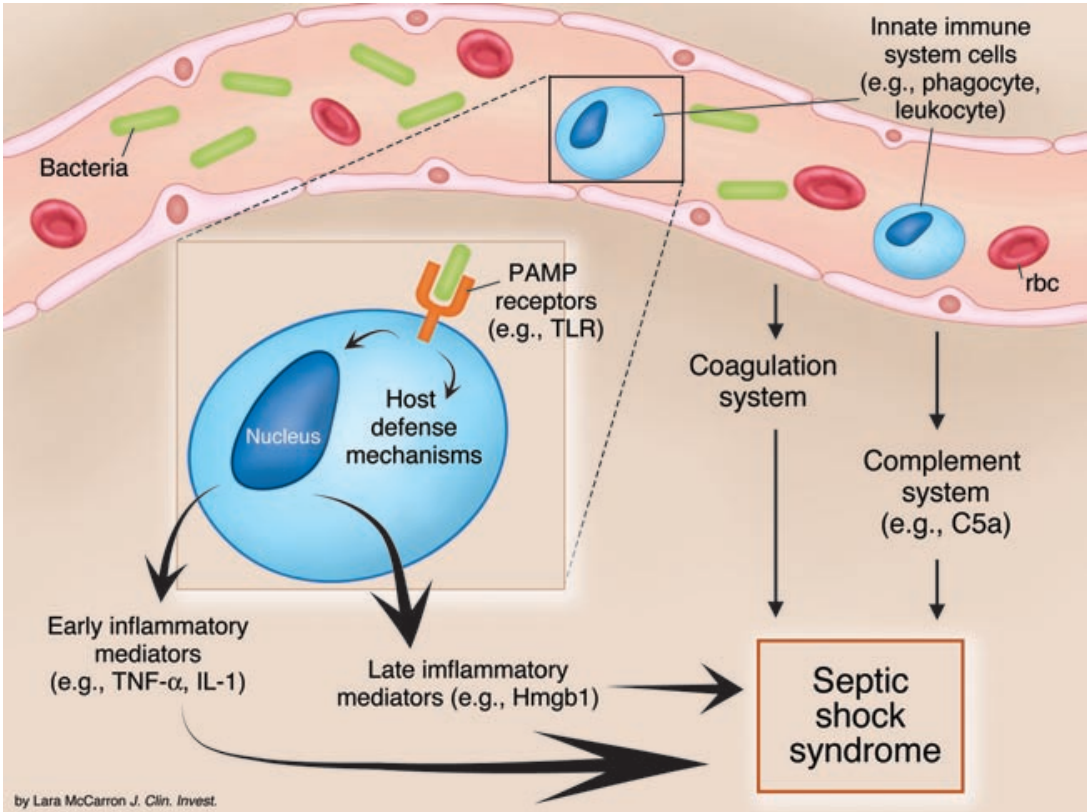

7 and 8 , and by the microbe DNA receptor TLR9, which creates a favorable environment for vaccination $(9-11)$. On the other hand, TLR-derived signals are also responsible for adverse effects of immune reactions like the septic shock syndrome (12). This is best illustrated by the fact that mice deficient either for TLR4, the receptor required for responses to the LPS of Gram-negative bacteria, or for essential transducers of TLR4-derived signals, are highly resistant to LPS infusion $(13,14)$. TLRs thus hold considerable promise for both agonistic and antagonistic therapeutic intervention.

\section{TLR2 blockade reduces the lethality of the septic shock syndrome in response to Gram-positive bacteria}

TLR2 interacts physically and functionally with TLR1 and TLR6 and mediates responses to Gram-positive bacteria and Mycobacteria, Borrelia (spirochetes), and yeast (15). Bacterial components stimulating TLR2 signaling are peptidoglycan, lipoteichoic acid, and tripalmitoylated lipoproteins (mimicked by the synthetic lipopeptide $\left.\mathrm{P}_{3} \mathrm{CSK}_{4}\right)(6,16)$. In their study, Meng and colleagues demonstrate the direct binding of $\mathrm{P}_{3} \mathrm{CSK}_{4}$ to the TLR2 ectodomain (4). They noted the ability of $\mathrm{P}_{3} \mathrm{CSK}_{4}$, or of viable and heat -inactivated Bacillus subtilis, to evoke a septic shock syndrome in mice that was strongly dependent on the presence of TLR2. Based on these observations the authors explored the ability of monoclonal antibody T2.5, raised in their laboratory against the TLR2 ectodomain, to suppress lethality in mouse models of the septic shock syndrome, caused by infection with Grampositive bacteria. Preparatory experiments clearly demonstrated the ability of this antibody to interfere with the formation of TLR $2 / \mathrm{P}_{3} \mathrm{CSK}_{4}$ complexes. Addition of T2.5 reduced macrophage signal transduction and synthesis of inflammatory cytokines after treatment with either $\mathrm{P}_{3} \mathrm{CSK}_{4}$ or B. subtilis. Similarly, pretreatment of mice with the antibody reduced serum concentrations of cytokines produced in response to $\mathrm{P}_{3} \mathrm{CSK}_{4}$ injection. Most of the TLR2 expressed in freshly isolated phagocytes was found in the cytoplasm. When the same cells were isolated from B. subtilis-infected mice, TLR2 cell surface expression was enhanced. This may indicate that less TLR 2 must be blocked at the onset of sepsis to prevent lethal shock.

One milligram of antibody T2.5 suppressed lethality in all experimental animals, both those presensitized with IFN- $\gamma$ and D-galactosamine before application of low-dose $\mathrm{P}_{3} \mathrm{CSK}_{4}$ and, alternatively, those treated directly with a high dose of heat-inactivated $B$. subtilis. In the case of the high-dose B. subtilis protocol, a therapeutic window for complete protection was defined as lasting from two hours prior to challenge until three hours after challenge. These results further estab-

\section{Figure 1}

Potential targets of treatments of septic shock. Interaction of bacteria with receptors recognizing PAMPs like the TLRs stimulates cells of the innate immune system to produce proinflammatory molecules, which, together with components of the activated complement and coagulation systems, promote the development of septic shock syndrome. Some prominent examples of molecules involved in the inflammatory cascade are shown in parentheses. lish the important role of TLRs in lethal inflammatory syndromes and pinpoint TLR2 as a target molecule for intervention in the harmful sequelae of infection with Gram-positive bacteria. They also prove the ability of antibodies to effectively disrupt the interaction of TLRs with their cognate ligands in vivo. It will be interesting to see whether similarly positive results can be obtained upon infection of mice with rapidly replicating viable bacteria.

Evaluations of the potential of TLR blockade for future clinical application must take a number of aspects into account. Limitations in the predictive value of rodent models for human disease are a frequent and unfortunate outcome and apply also to the therapy of septic shock. For example, antibodies to LPS, as well as TNF- $\alpha$ or IL- 1 antagonists, protected mice from lethal septic shock syndrome but have so far proven ineffective in human clinical trials (3). The presentation by bacteria of ligands for multiple TLRs may necessitate simultaneous treatment with several antagonists $(17,18)$. Furthermore, the extent of the therapeutic window for each intervention strategy will most likely be of critical importance. In this regard the effectiveness of various drugs that interfere with crucial early events of the inflammatory cascade like TLR occupancy must be compared. Alternative strategies aimed at the inhibition of late inflammatory mediators might 
increase the time span during which successful treatment is possible. In this regard the results of animal experiments blocking the activity of the high-mobility group box 1 protein (Hmgb1), a late mediator of the endotoxin-induced septic shock syndrome, are encouraging (19). The inhibition of late inflammatory mediators may rival TLR antagonism as an equally promising new approach for the clinical treatment of the septic shock syndrome.

\section{Acknowledgments}

Work in the author's laboratory is supported by the Austrian Science Foundation (Fonds zur Förderung der Wissenschaftlichen Forschung grant P15272). Critical reading of the manuscript by Manuela Baccarini and Rodger Novak and the design of Figure 1 by Manuela Baccarini are gratefully acknowledged.

Address correspondence to: Thomas Decker, Max F. Perutz Laboratories, University Departments at the Vienna Biocenter, Department of Microbiology and Genetics, University of Vienna, Dr. Bohr-Gasse
9/4, A1030 Vienna, Austria. Phone: 43-14277-54605; Fax: 43-1-4277-9546; E-mail: thomas.decker@univie.ac.at.

1. Riedemann, N.C., Guo, R.F., and Ward, P.A. 2003. The enigma of sepsis. J. Clin. Invest. 112:460-467. doi:10.1172/JCI200319523.

2. Angus, D.C., et al. 2001. Epidemiology of severe sepsis in the United States: analysis of incidence, outcome, and associated costs of care. Crit. Care Med. 29:1303-1310

3. Riedemann, N.C., Guo, R.F., and Ward, P.A. 2003. Novel strategies for the treatment of sepsis. Nat. Med. 9:517-524.

4. Meng, G., et al. 2004. Antagonistic antibody prevents toll-like receptor 2 -driven lethal shocklike syndromes. J. Clin. Invest. 113:1473-1481. doi:10.1172/JCI200420762.

5. Medzhitov, R., and Janeway, C.A., Jr. 1997. Innate immunity: the virtues of a nonclonal system of recognition. Cell. 91:295-298.

6. Takeda, K., Kaisho, T., and Akira, S. 2003. Toll-like receptors. Annu. Rev. Immunol. 21:335-376.

7. Zhang, D., et al. 2004. A toll-like receptor that prevents infection by uropathogenic bacteria. Science. 303:1522-1526.

8. Hawn, T.R., et al. 2003. A common dominant TLR5 stop codon polymorphism abolishes flagellin signaling and is associated with susceptibility to legionnaires' disease. J. Exp. Med. 198:1563-1572.

9. Akira, S., and Hemmi, H. 2003. Recognition of pathogen-associated molecular patterns by TLR family. Immunol. Lett. 85:85-95.

10. Jurk, M., et al. 2002. Human TLR7 or TLR8 inde- pendently confer responsiveness to the antiviral compound R-848. Nat. Immunol. 3:499.

11. Bauer, S., and Wagner, H. 2002. Bacterial CpGDNA licenses TLR9. Curr. Top. Microbiol. Immunol. 270:145-154.

12. Lorenz, E., Mira, J.P., Frees, K.L., and Schwartz, D.A. 2002. Relevance of mutations in the TLR4 receptor in patients with gram-negative septic shock. Arch. Intern. Med. 162:1028-1032.

13. Kawai, T., Adachi, O., Ogawa, T., Takeda, K., and Akira, S. 1999. Unresponsiveness of MyD88-deficient mice to endotoxin. Immunity. 11:115-122.

14. Takeuchi, O., et al. Differential roles of TLR2 and TLR4 in recognition of gram-negative and grampositive bacterial cell wall components. Immunity. 11:443-451.

15. Ozinsky, A., et al. 2000. The repertoire for pattern recognition of pathogens by the innate immune system is defined by cooperation between Toll-like receptors. Proc. Natl. Acad. Sci. U. S. A. 97:13766-13771.

16. Kirschning, C.J., and Schumann, R.R. 2002. TLR2: cellular sensor for microbial and endogenous molecular patterns. Curr. Top. Microbiol. Immunol. 270:121-144.

17. Nau, G.J., Schlesinger, A., Richmond, J.F., and Young, R.A. 2003. Cumulative Toll-like receptor activation in human macrophages treated with whole bacteria. J. Immunol. 170:5203-5209.

18. Weiss, D.S., Raupach, B., Takeda, K., Akira, S., and Zychlinsky, A. 2004. Toll-like receptors are temporally involved in host defense. J. Immunol. 172:4463-4469.

19. Wang, H., et al. 1999. HMG-1 as a late mediator of endotoxin lethality in mice. Science. 285:248-251. 